\title{
24-h urinary free cortisol from mid-pregnancy to 3-months postpartum: Gender and parity differences and effects
}

\author{
Ana Conde $^{\mathrm{a}, \mathrm{b}, *}$, Bárbara Figueiredo $^{\mathrm{b}}$ \\ a Portucalense Infante D. Henrique University, Porto, Portugal \\ b School of Psychology, University of Minho, Braga, Portugal
}

Received 17 January 2014; received in revised form 18 August 2014; accepted 25 August 2014

\author{
KEYWORDS \\ Cortisol; \\ Pregnancy; \\ Postpartum; \\ Women/men; \\ 1st/2nd time parents
}

\begin{abstract}
Summary
Background: Pregnancy and postpartum have been associated to several physiological changes; however, empirical evidence was almost exclusively obtained in primiparous women and few studies focus on hormonal changes in men and second-time parents. The main aim of this study is to examine 24-h urinary free cortisol from mid-pregnancy to 3-months postpartum, comparing women/men and first/second-time parents.

Methods: Twenty-six women and 22 men $(N=48)$ were recruited from an antenatal obstetric unit in Porto, Portugal. 24-h urinary free cortisol was measured at the 2 nd and 3rd trimester and at 3-months postpartum. Repeated measures analyses of variance were conducted, in order to analyze 24-h urinary free cortisol patterns of change over this period. Gender and parity were included in the analyses as potential modifiers, in order to compare women and men, and firstand second-time parents.

Results: An increase from the 2 nd to the $3 r d$ trimester $(p=.006)$ and a decrease from the 3 rd trimester to 3 -months postpartum $(p=.005)$ were reported in all parents' 24 -h urinary free cortisol. The interaction effects for Time * Gender $(p=.03)$ and Time *Parity $(p=.02)$ were found. Women and first-time parents revealed higher levels, while men and second-time parents showed lower 24-h urinary free cortisol levels at the 2nd trimester than at 3-months postpartum. Conclusions: Findings appear to clarify the direction, as well as, the timing, gender and parity extension of 24-h urinary free cortisol changes from mid-pregnancy to 3-months postpartum. The same pattern of change in all parents' 24-h urinary free cortisol from mid-pregnancy to 3months postpartum is consistent with the proposed role of hormones in preparation to parenting.
\end{abstract}

\footnotetext{
* Corresponding author at: Portucalense Infante D. Henrique University, Porto, Portugal. Tel.: +35196 4535741.

E-mail address: anac@upt.pt (A. Conde).
} 
Gender and parity differences and effects on 24-h urinary free cortisol are also consistent with cortisol as a stress biomarker for higher challenges associated to pregnancy and childbirth in women and first-time parents versus higher demands related to after childbirth parenting in men and second-time parents.

(c) 2014 Elsevier Ltd. All rights reserved.

\section{Introduction}

Pregnancy and postpartum have been associated to several physiological changes, namely the dysregulation of the hypothalamic-pituitary-adrenal axis (HPA) and the autonomic nervous system (ANS) functioning (Brunton et al., 2008; Lazinski et al., 2008; O’Keane et al., 2011; Duthie and Reynolds, 2013). However, empirical evidence was almost exclusively obtained in primiparous women and few studies focus on hormonal changes in men and second-time parents.

Theoretical approaches concerning HPA axis functioning during pregnancy and postpartum have mainly assumed two pathways. The HPA axis was considered an important system involved in the regulation of parental behavior (Carter and Porges, 2012; Gonzalez et al., 2009). The HPA axis was also believed to play a fundamental role in the response to external and internal stimuli and to provide a regulatory network linking the brain with the body's behavioral and physiological responses to stress (Dipietro, 2012; Reynolds et al., 2013). Both paths will subsequently be analyzed in further detail.

Research on human parenting psychobiology is receiving new attention. An association between the HPA axis activity and the subsequent expression of social behaviors and bonds has been proposed. Positive social behaviors, including social bonds, may reduce HPA axis activity, while negative social interactions can have the opposite effect (for a revision see Carter, 1998). Findings revealed a decrease in cortisol with pair-bond formation (Ziegler et al., 1996; Carter, 1998; Reburn and Wynne-Edwards, 1999) and higher cortisol levels are significantly and strongly associated with maternal approach behaviors and positive maternal attitudes (Fleming et al., 1997; Stallings et al., 2001). While some reference was made to relevant research on men, the emphasis on women continues to reflect the state of art on physiological influences in terms of parental behavior. Focus on both women and men's hormonal changes to establish patterns of changes over pregnancy and postpartum is relevant to fully understand the psychobiology of parenthood.

The HPA axis has also been pointed out as the primary biological mechanism underlying stress regulation and adaptation (Dipietro, 2012; Reynolds et al., 2013). Changes in the HPA axis, mainly in the postpartum period, were associated to mood disturbances and several puerperal disorders (Corwin and Pajer, 2008; Meinlschmidt et al., 2010; O'Keane et al., 2011). Considered as the end product of the HPA axis, cortisol has been viewed as a biomarker of psychological stress (Harville et al., 2007). Some studies associate high-cortisol levels to psychological symptoms during pregnancy and the postpartum period (Field et al., 2004; Obel et al., 2005), while others show that stress and anxiety levels assessed by self-report measures do not necessarily correlate with maternal cortisol levels (Harville et al., 2009; Voegtline et al., 2013).

\subsection{Changes in cortisol during pregnancy and the postpartum period}

Findings from previous research suggest that levels of cortisol generally increase as pregnancy progresses (Obel et al., 2005; Jones et al., 2006; D’Anna-Hernandez et al., 2011), reaching peak levels during the third trimester (Jung et al., 2011). After childbirth, maternal cortisol levels were usually reported to decrease (Field et al., 2004; de Weerth and Buitelaar, 2005; Figueiredo and Costa, 2009; Cheng and Pickler, 2010). Nevertheless, conflicting results depended on the type of cortisol matrices used. Jung et al. (2011), for example, observed that total plasma cortisol and cortisteroid-binding globulin remained high at 2-3 months postpartum, whereas urinary free cortisol and plasma free cortisol returned to baseline level, and mean total cortisol concentrations were lower after childbirth compared with pregnancy.

Cortisol changes during pregnancy and postpartum were analyzed with a variety of matrices and different assessment time points. Little is known about 24-h urinary free cortisol pattern of changes over pregnancy and postpartum, as proposed by the present study.

\subsection{Gender differences in cortisol levels}

Empirical research on HPA axis sex differences has produce inconsistent or even contradictory findings, especially in humans (for a revision see Kudielka and Kirschbaum, 2005). The sample size, composition in relation to health status, age at testing, the type of stressor/challenge and the duration of stress exposure are some of the proposed factors to explain conflicting results (for a revision see Kudielka and Kirschbaum, 2005). Men and women with similar stage-specific differences in hormone levels, including higher concentrations in cortisol in the period just before birth, were suggested (Storey et al., 2000). In women these changes result, at least, partially from the secretion of corticotropin-releasing hormone $(\mathrm{CRH})$ from the placenta (Magiakow et al., 1996; Mastorakos and Ilias, 2003), stimulated by maternal cortisol and a positive-feed forward mechanism (Mastorakos and Ilias, 2003). Less is known about the basis of such changes in men. Recent studies put forward the hypothesis that the underlying neuroendocrine control of mammalian parental behavior may be homologous in males and females (for a revision see Wynne-Edwards and Reburn, 2000; Nunes-Costa et al., 2015). The study of gender differences and effects in 24-h urinary free cortisol levels and changes from mid-pregnancy to 3-months postpartum can bring further empirical evidence to this hypothesis. 


\subsection{Parity differences in cortisol levels}

Higher cortisol levels in both primiparous and multiparous mothers were associated with behavioral activation. However, the association between cortisol levels and mothering behavior seems to be moderated by parity, as only multiparous cortisol was correlated with instrumental caregiving activities, as oppose to producing affectionate behaviors (Fleming et al., 1997).

Parity seems to be an important variable to take into consideration in cortisol reactivity to psychological stressors during the pregnancy/lactation period (e.g., Tu et al., 2006). The study of parity differences and effects on 24-h urinary free cortisol levels and changes from mid-pregnancy to 3-months postpartum may reflect different patterns of psychological adjustment during pregnancy and the postpartum period in first- and second-time parents (e.g., Skari et al., 2002; Figueiredo and Conde, 2011). However, some studies did not found differences concerning parity in both prenatal and postnatal cortisol reactivity (e.g., Cheng and Pickler, 2010).

Previous studies about cortisol during pregnancy and postpartum mainly focused on primiparous women, analyzing cortisol reactivity under stressful situations. Little is known about cortisol changes in men and in second-time parents and involving naturalistic, longitudinal designs.

In order to contribute towards the further understanding of physiological changes in the HPA axis during pregnancy and postpartum, the present research aims to examine 24-h urinary free cortisol from mid-pregnancy to 3-months postpartum. An increase from the 2 nd to the 3rd trimester and a decrease from the $3 \mathrm{rd}$ trimester to 3-months postpartum in all parents' 24-h urinary free cortisol was hypothesized and based on previous empirical evidence related with cortisol levels over this period (e.g., Jones et al., 2006; Figueiredo and Costa, 2009; Cheng and Pickler, 2010; D’Anna-Hernandez et al., 2011; Jung et al., 2011).

The present study also aims to explore gender and parity differences and effects in 24-h urinary free cortisol levels and changes from mid-pregnancy to 3-months postpartum. Higher 24-h urinary free cortisol levels in women and first-time parents versus lower levels reported in men and second-time parents at the 2 nd trimester than at the 3-months postpartum period was also hypothesized. It was based on existing literature on gender, parity and psychological adjustment during pregnancy and postpartum (e.g., Skari et al., 2002; Figueiredo and Conde, 2011).

\section{Methods}

\subsection{Participants}

A sample of 26 women and 22 men $(N=48)$, with no identified psychosocial or medical risk, was recruited from an antenatal obstetric unit in Porto, Portugal. Inclusion criteria were: read and write Portuguese, single gestation, no medical/obstetric risk, and 24-h urinary free cortisol measures at the 2nd and 3rd trimesters and at 3months postpartum. Nearly all participants were Portuguese (83.3\%) and lived with a partner (and children) $\mathbf{( 9 5 . 8 \% ) .}$ The vast majority was between 20 and 29 (66.7\%); had low or medium-low socio-economical background (61.3\%); and were employed (93.8\%). About $65 \%$ of participants were firsttime parents and $35.4 \%$ second-time parents (see Table 1). Socio-demographic features (Santos et al., 2011) and the prevalence of other relevant conditions, such as anxiety and depression (Teixeira et al., 2009; Figueiredo and Conde, 2011), are similar to other studies developed with the Portuguese low risk obstetric population. No gender differences were found for any of considered demographic variables. Parity differences were found for education $\left[\chi^{2}(2)=11.45\right.$, $p=.003]$.

\subsection{Procedure}

The present research was conducted in accordance with the Helsinki Declaration. After approval by the review board of the institution, participants were invited to participate in the study at their first medical appointment (prior 14 weeks' gestation) at the antenatal obstetric unit. The aims and the procedures of the study were explained. After signing an informed consent form, the socio-demographic questionnaire was separately filled by the pregnant women and the men. The participants collected $24-\mathrm{h}$ urine samples at the 2nd $(M=20.5$ weeks gestation, $S D=.94)$ and 3rd pregnancy trimester $(M=31.6$ weeks gestation, $S D=2.6)$ and at 3-months postpartum.

\subsection{Measures (women and men)}

\subsubsection{Socio-demographic questionnaire}

Information about the participants (e.g., age, occupational status, marital status) was collected using a sociodemographic questionnaire (Teixeira et al., 2009).

\subsubsection{4-h urinary free cortisol}

Research on cortisol involves a variety of matrices. Many studies include single blood samples (D'Anna-Hernandez et al., 2011), while others, especially based on naturalistic longitudinal designs, involving the collection of multiple bio specimens per participant, privilege urine and saliva samples over other matrices (Sarkar et al., 2013). Saliva, plasma and 24-h urinary free cortisol are not interchangeable, as they provide different kinds of information about ambient HPA activity (Yehuda et al., 2003). Since saliva cortisol is a response mechanism to acute conditions (Kirschbaum and Hellhammer, 1994), even repeated measurements may not be the most informative way to evaluate overall or longterm HPA activity, as during the prenatal and postnatal period. A measure of cortisol over a longer period of time is being suggested as a more useful practice to evaluate the overall HPA activity in non-acute experimental situations (D’Anna-Hernandez et al., 2011). An integrated mean of cortisol levels over 24 -h period is thought to provide a moderately stable measure of adrenocortical output, particularly when participants collect urine on days where they can restrict environmental exposure to stressors, or other elicitors of the HPA axis (Yehuda et al. , 2003). Moreover, 24-h urinary free cortisol enables us to control the effect of time of the day in which participants collected urine samples, which is associated with a high variability in cortisol levels. Since we are not interested in cortisol response in acute 
Table 1 Socio-demographics (women and men, first and second-time parents, and total sample).

\begin{tabular}{|c|c|c|c|c|c|}
\hline & $\begin{array}{l}\text { Women } \\
n=26 \\
\%\end{array}$ & $\begin{array}{l}\text { Men } \\
n=22 \\
\%\end{array}$ & $\begin{array}{l}\text { 1st time } \\
n=31 \\
\%\end{array}$ & $\begin{array}{l}\text { 2nd time } \\
n=17 \\
\%\end{array}$ & $\begin{array}{l}\text { Total } \\
N=48 \\
\%\end{array}$ \\
\hline \multicolumn{6}{|l|}{ Age (years) } \\
\hline$[20-29]$ & 30.8 & 27.3 & 32.3 & 23.5 & 29.2 \\
\hline$[30-39]$ & 69.2 & 63.6 & 64.5 & 70.6 & 66.6 \\
\hline$[40-49]$ & 0.0 & 9.1 & 3.2 & 5.9 & 4.2 \\
\hline \multicolumn{6}{|l|}{ Matrimonial status } \\
\hline Cohabiting & 19.2 & 31.8 & 29.0 & 17.6 & 25.0 \\
\hline Married & 80.8 & 68.2 & 71.0 & 82.4 & 75.0 \\
\hline \multicolumn{6}{|l|}{ Occupational status } \\
\hline Employed & 92.3 & 95.5 & 96.8 & 88.2 & 93.8 \\
\hline Unemployed & 3.8 & 4.5 & 3.2 & 5.9 & 4.2 \\
\hline Student & 3.9 & 0.0 & 0.0 & 5.9 & 2.1 \\
\hline \multicolumn{6}{|l|}{ Profession } \\
\hline Non manual qualified & 33.3 & 25.0 & 27.3 & 33.3 & 29.0 \\
\hline Non manual not qualified & 0.0 & 18.8 & 9.1 & 11.1 & 9.7 \\
\hline Manual qualified & 20.0 & 43.7 & 31.8 & 33.4 & 32.3 \\
\hline Manual not qualified & 46.7 & 12.5 & 31.8 & 22.2 & 29.0 \\
\hline \multicolumn{6}{|l|}{ Schooling (years) } \\
\hline$<9$ & 19.2 & 31.8 & 9.7 & 52.9 & 25.0 \\
\hline [9-12] & 30.8 & 45.5 & 48.4 & 17.7 & 37.5 \\
\hline$>12$ & 50.0 & 22.7 & 41.9 & 29.4 & 37.5 \\
\hline
\end{tabular}

situations, but rather in the evaluation of overall or longterm HPA activity during the prenatal and early postnatal period in women, men, first- or second-time parents, 24-h free cortisol was selected as an index of the total amount of cortisol released by the adrenals over a complete circadian cycle, which is considered a physiologically meaningful unity of time. Among the general population, the reference range of 24-h urinary free cortisol levels is $55.5-286.0 \mu \mathrm{g} / 24 \mathrm{~h}$ (Bayer Diagnostics ADVIA Centaur Reference Manual).

Participants were asked to collect all urine produced during a 24-h period according to the following instructions: (1) registration of the day and time of the first urine collection, (2) collection and storage the urine produced in a cool and dark place, and (3) collection the last urine sample on the following day at the same time of the initial collection. For assay, total volume of urine produced during a 24-h period was registered. The urine samples were kept frozen at $-20^{\circ} \mathrm{C}$ and then analyzed in laboratory, using the cortisol ADVIA Centaur immunoassay system (Bayer Diagnostics). This system consists of a competitive immunoassay using direct chemiluminescent technology. The procedure followed: (1) take a $20 \mathrm{~nL}$ sample; (2) add $50 \mathrm{~nL}$ of Lite reagent and $250 \mathrm{~nL}$ of solid phase and incubate at $37^{\circ} \mathrm{C}$ for $5 \mathrm{~min}$; (3) separate, aspirate and wash the recipient with reagent water; (4) add the same amount of acid and basic reagent to initiate the chemiluminescent reaction (Bayer Diagnostics ADVIA Centaur Assay Manual). Total volumes with less than $750 \mathrm{~mL}$ of urine were excluded from lab analysis, since they do not allow a valid determination of hormone measurement. Continuous levels were used in statistical analysis.

\subsection{Statistical analysis}

Repeated measures analyses of variance were conducted in order to study 24-h urinary free cortisol patterns over the 2nd and 3rd pregnancy trimesters and 3-months postpartum. Preliminary analyses of variance to select potential confounders were implemented. No significant differences were found in 24-h urinary free cortisol at the 2 nd and 3 rd trimester or at 3-months postpartum, concerning age, matrimonial and occupational status, profession or schooling. Significant/marginally significant differences were found concerning gender (cortisol levels at the 2nd trimester: $t(46)=1.80, p=.08$; 3rd trimester: $t(46)=1.80, p=.02$ ) and parity (cortisol levels at the 3rd trimester: $t(46)=1.71$, $p=.09)$. Gender and parity were included in repeated measures analyses of variance as potential modifiers, in order to compare women and men and first- and second-time parents. In the construction of the model, 24-h urinary free cortisol levels were included as measures of the same within-subject factor with three levels, corresponding to the three measuring time points (2nd and 3rd pregnancy trimesters and 3-months postpartum). Gender and parity were considered between-subjects factors of the analysis. Main effects and interaction terms were tested. Post hoc pair-wise comparisons with the Bonferroni correction were applied to analyze mean differences among measuring time points. Independent repeated measures ANOVAs were used to study $24-\mathrm{h}$ urinary free cortisol patterns from midpregnancy to 3-months postpartum, respectively in women, men, and first- and second-time parents. Bifactorial ANOVAs were performed to test gender and parity differences in 
Table 2 24-h urinary free cortisol $(\mu \mathrm{g} / 24 \mathrm{~h})$ from mid-pregnancy to 3-months postpartum: men $(n=22)$ and women $(n=26)$, first $(n=31)$ and second $(n=17)$ time parents, and total sample $(N=48)$.

\begin{tabular}{llll}
\hline & $\begin{array}{l}\text { 2nd } \\
\text { trimester } \\
M(S D)\end{array}$ & $\begin{array}{l}\text { 3rd } \\
\text { trimester } \\
M(S D)\end{array}$ & $\begin{array}{l}\text { 3-Months } \\
\text { postpartum } \\
M(S D)\end{array}$ \\
\hline $\begin{array}{l}\text { Men } \\
\text { 1st time }\end{array}$ & $93.00(35.80)$ & $66.41(25.78)$ \\
2nd time & $67.67(25.25)$ & $76.28(15.24)$ & $93.14(49.83)$ \\
Total & $72.18(26.03)$ & $86.92(30.63)$ & $76.13(37.58)$ \\
Women & $69.31(25.01)$ & & \\
1st time & & $120.15(42.56)$ & $72.06(43.19)$ \\
2nd time & $89.90(36.66)$ & $99.48(34.77)$ & $74.56(34.08)$ \\
Total & $75.11(23.48)$ & $113.00(40.58)$ & $72.92(39.58)$ \\
Total & $84.78(32.99)$ & & \\
1st time & & $107.89(41.36)$ & $69.51(35.93)$ \\
2nd time & & $88.56(29.13)$ & $83.30(41.94)$ \\
Total & $79.86(33.46)$ & $101.04(38.31)$ & $74.39(38.30)$ \\
\hline
\end{tabular}

24-h urinary free cortisol levels at each measuring time points. Partial eta-squared $\left(\eta_{\mathrm{p}}^{2}\right)$ was used as a measure of effect size. Its interpretation was done according to the rule of thumb (Cohen) $(.01 \sim$ small; $.06 \sim$ medium; $.14 \sim$ large).

\section{Results}

\subsection{4-h urinary free cortisol changes from mid-pregnancy to 3-months postpartum}

When total sample was considered, repeated measures ANOVA revealed significant changes in 24-h urinary free cortisol levels from mid-pregnancy to 3-months postpartum $\left[F(2,88)=6.01, p=.004, \eta_{\mathrm{p}}^{2}=.120\right]$. Pair wise comparisons concerning 24-h urinary free cortisol levels revealed significant mean differences $(M D)$ between the 2 nd and the $3 r d$ pregnancy trimester $(M D=-21.01 ; p=.02)$ and between the 3rd pregnancy trimester and 3-months postpartum $(M D=20.68 ; p=.02)$, but not between the 2nd pregnancy trimester and 3-months postpartum $(M D=-.32 ; p=1.00)$ (see Table 2).

\subsection{Gender differences and effects in 24-h urinary free cortisol levels and changes from mid-pregnancy to 3-months postpartum}

When total sample was considered, repeated measures ANOVA did not reveal significant gender effects in 24-h urinary free cortisol levels $\left[F(1,44)=2.29, p=.14, \eta_{p}^{2}=.050\right]$. When differences between men and women were explored at each measuring time points, significant lower 24-h urinary free cortisol levels were found in men than in women at the 3 rd pregnancy trimester $[F(1,44)=5.35, p=.02]$, but no significant differences were found either at the 2nd pregnancy trimester $[F(1,44)=1.95, p=.17]$ or at 3-months postpartum $[F(1,44)=.31, p=.58]$ (see Table 2$)$.

The interaction effect for Time * Gender in 24-h urinary free cortisol levels was significant between the 2 nd pregnancy trimester and 3-months postpartum $[F(1,44)=5.04$, $\left.p=.03, \eta_{\mathrm{p}}^{2}=.103\right]$, but not between the $2 \mathrm{nd}$ and the $3 \mathrm{rd}$ pregnancy trimesters $\left[F(1,44)=.74, p=.39, \eta_{\mathrm{p}}^{2}=.017\right]$ : while for men higher 24 -h free cortisol levels were found at 3-months postpartum than at the 2 nd trimester, in women higher cortisol levels were found at the 2nd trimester than at 3-months postpartum (see Fig. 1).

Pair wise comparisons concerning men's 24-h urinary free cortisol levels did not reveal significant mean differences either between the 2 nd and the 3 rd pregnancy trimesters $(M D=-14.71 ; p=.24)$, or between the 3rd pregnancy trimester and 3-months postpartum $(M D=4.86 ; p=1.00)$ or between the 2 nd pregnancy trimester and 3-months postpartum $(M D=-9.85 ; p=.70)$. Pair wise comparisons concerning women's 24-h urinary free cortisol levels revealed significant mean differences between the 3rd pregnancy trimester and

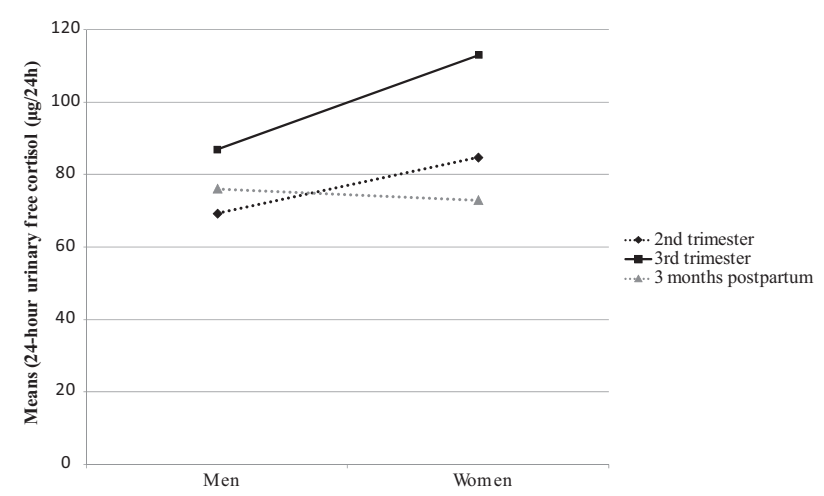

Figure 1 Time * Gender interaction for 24-h urinary free cortisol. 


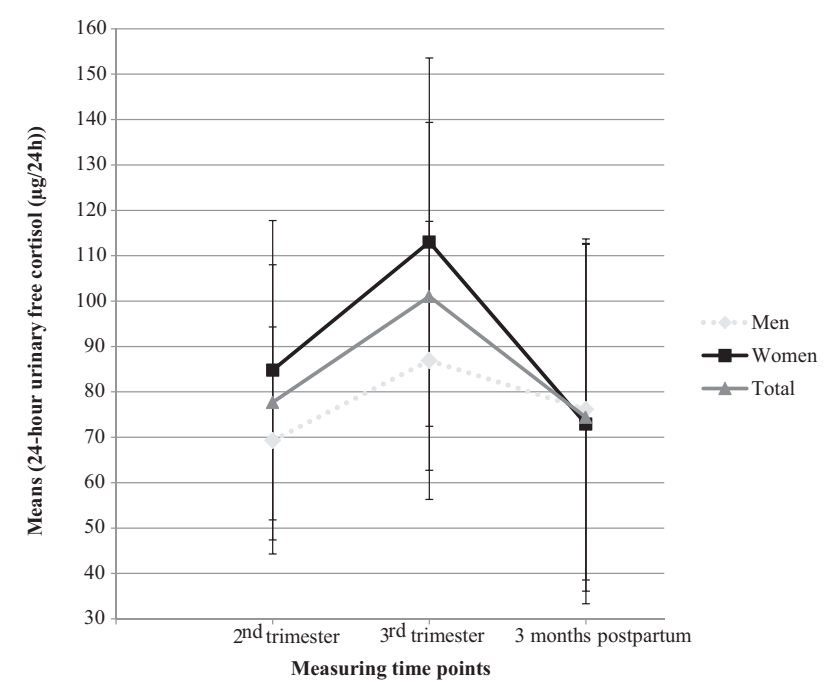

Figure 2 24-h urinary free cortisol $(\mu \mathrm{g} / 24 \mathrm{~h})$ from midpregnancy to 3-months postpartum: men $(n=22)$, women $(n=26)$ and total sample $(N=48)$.

3-months postpartum $(M D=36.51 ; p=.01)$, but not between the $2 \mathrm{nd}$ and the 3rd pregnancy trimester $(M D=-27.31$; $p=.08$ ) or between the 2 nd pregnancy trimester and 3months postpartum $(M D=9.20 ; p=1.00)$ (see Fig. 2).

\subsection{Parity differences and effects in 24-h urinary free cortisol levels and changes from mid-pregnancy to 3-months postpartum}

When total sample was considered, repeated measures ANOVA did not show significant parity effects in 24-h urinary free cortisol levels $\left[F(1,44)=.20, p=.66, \eta_{p}^{2}=.005\right]$. When differences between first- and second-time parents were examined at each of measuring time points, no significant differences were found either at the 2nd pregnancy trimester $[F(1,44)=.33, p=.57]$, at the 3rd pregnancy trimester $[F(1,44)=2.95, p=.09]$ or at 3-months postpartum $[F(1,44)=1.57, p=.22]$ (see Table 2 ).

The interaction effect for Time * Parity in 24-h urinary free cortisol levels was significant between the 2nd pregnancy trimester and 3-months postpartum $[F(1,44)=5.58$, $\left.p=.02, \eta_{\mathrm{p}}^{2}=.113\right]$, but not between the $2 \mathrm{nd}$ and the $3 \mathrm{rd}$ pregnancy trimesters $\left[F(1,44)=.86, p=.36, \eta_{\mathrm{p}}^{2}=.019\right]$ : while for second-time parents higher 24 -h free cortisol levels were found at 3-months postpartum than at the 2nd trimester, in first-time parents higher cortisol levels were found at the 2 nd trimester than at 3-months postpartum (see Fig. 3).

Pair wise comparisons concerning first-time parents' 24-h urinary free cortisol levels revealed significant mean differences between the 2nd and the 3rd pregnancy trimesters $(M D=-27.79 ; p=.02)$ and the 3rd pregnancy trimester and 3-months postpartum $(M D=37.34 ; p=.000)$, but not between the 2 nd pregnancy trimester and 3-months postpartum $(M D=9.55 ; p=.69)$. Pair wise comparisons concerning second-time parents' 24-h urinary free cortisol did not show significant mean differences between the 2nd and the 3rd pregnancy trimesters $(M D=-14.23 ; p=.50)$, the $3 r d$

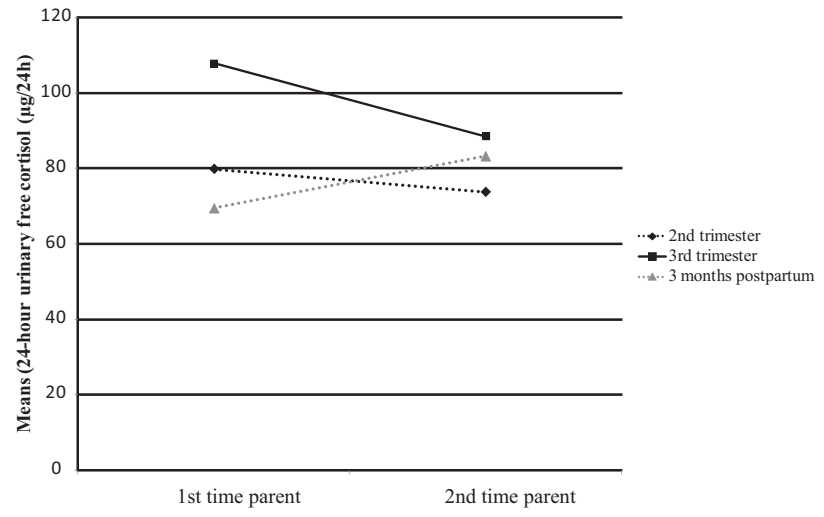

Figure 3 Time * Parity interaction for 24-h urinary free cortisol.

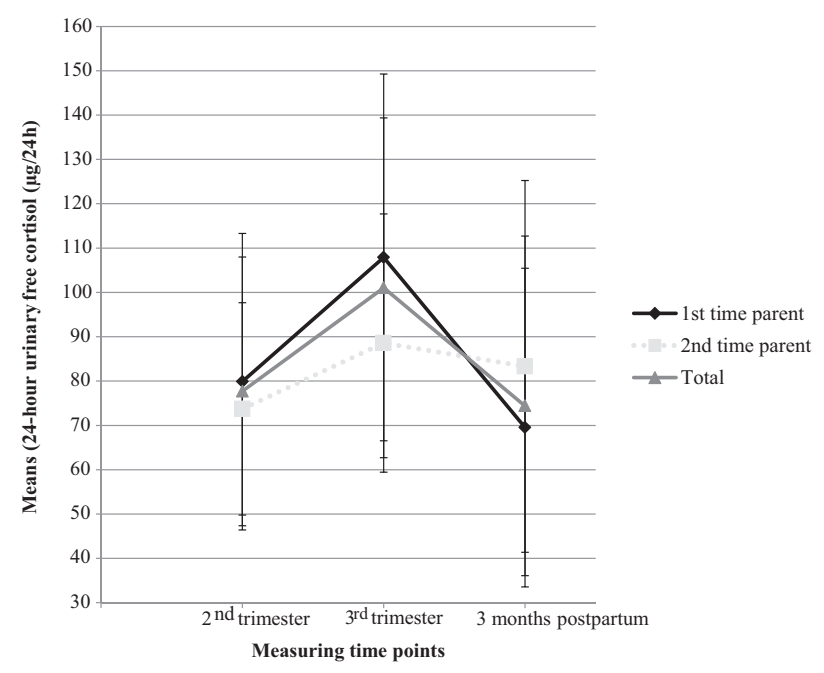

Figure 4 24-h urinary free cortisol $(\mu \mathrm{g} / 24 \mathrm{~h})$ from midpregnancy to 3-months postpartum: first $(n=31)$ and second $(n=17)$ time parents, and total sample $(N=48)$.

pregnancy trimester and 3-months postpartum $(M D=4.03$; $p=1.00$ ) or between the 2nd pregnancy trimester and 3months postpartum $(M D=-10.20 ; p=.98)$ (see Fig. 4).

The interaction effect for Time * Gender * Parity in 24$\mathrm{h}$ urinary free cortisol levels was non-significant $[F(2$, 88) $\left.=.29, p=.75, \eta_{\mathrm{p}}^{2}=.007\right]$. The interaction effect for Gender*Parity in 24-h urinary free cortisol levels was also non-significant neither in repeated measures ANOVA [ $F(1$, $44)=1.32, p=.26]$, nor when the 2 nd $[F(1,44)=1.15$, $p=.29]$, 3rd trimesters $[F(1,44)=.03, p=.86]$ and 3 -months postpartum $[F(1,44)=1.08, p=.30]$ were independently considered.

\section{Discussion}

Changes in 24-h urinary free cortisol levels from midpregnancy to 3-months postpartum were found in all parents, with an increase from the 2 nd to the 3rd pregnancy trimester and a decrease from the 3rd pregnancy trimester to 3-months postpartum. This is a trend found in the literature, where a rise in cortisol levels during pregnancy and a decrease at early postpartum were consistently reported 
(de Weerth and Buitelaar, 2005; Obel et al., 2005; Figueiredo and Costa, 2009; Jung et al., 2011).

Overall, the same pattern of change in all parents' 24-h urinary free cortisol from mid-pregnancy to 3-months postpartum is consistent with the role of hormonal changes in gestation and preparation to parenting (Carter and Porges, 2012). Several suggestions can be found in literature about this pattern in women. The increment in cortisol levels as pregnancy progresses was considered important for foetal development, as maternal cortisol affects foetal hormone levels and placental structure and perfusion (Jensen et al., 2005). Other authors suggest that the increase of cortisol during pregnancy is related to circulating $\mathrm{CRH}$ concentrations critical for the initiation of the parturition (O’Keane et al., 2011). Jung et al. (2011) propose the rise in plasma free cortisol during pregnancy as an indicator of the maternal HPA axis across this period. The greater increase in urinary free cortisol also associated with pregnancy reflect the increase in both cortisol production and clearance. On the other hand, the decrease in cortisol levels observed at postpartum support the notion that the HPA axis gradually recovers during the postpartum period from its activated state during pregnancy (Mastorakos and Ilias, 2003; Kammerer et al. , 2006; Jung et al., 2011). The HPA axis remains relatively hypo responsive during the postpartum period, probably as a consequence of an adrenal hypertrophy during pregnancy, high rates of cortisol non-suppression to dexamethasone present at the first three postpartum weeks (Owens et al., 1987) and other inhibitory brain factors, such us oxytocin or prolactin (O'Keane et al., 2011). Since these explanations are mostly based in phenomena related to gestation, parturition and lactation, they cannot be applied to men. A similar pattern of change in 24-h urinary free cortisol from mid-pregnancy to 3-months postpartum between women and men is consistent with results indicating that men and women may have similar stagespecific differences in hormone levels (Storey et al., 2000) and with findings suggesting that underlying neuroendocrine control of parental behavior may be homologous in both men and women (for a review see Wynne-Edwards and Reburn, 2000; Nunes-Costa et al., 2015). This finding need to be replicated and future research should therefore maintain men as the focus of attention to enable a deeper understanding of the meaning and mechanisms of cortisol changes observed during this period.

Higher 24-h urinary free cortisol levels were observed in women compared to men at the 3rd pregnancy trimester, with no significant differences being observed neither at the 2nd trimester nor at 3-months postpartum. This may reflect the importance of cortisol in the initiation of the parturition (O'Keane et al., 2011) and acknowledge the need for this hypothesis to be fully tested in future research.

An interaction effect for Time* Gender and for Time* Parity was also reported in the present study. Women and first-time parents showed higher, while men and second-time parents registered lower 24-h urinary free cortisol levels at the 2nd trimester than at 3-months postpartum. The increase observed in cortisol levels from the 2 nd to the 3rd pregnancy trimester and the decrease occurring from the 3rd pregnancy trimester to 3-months postpartum is more prominent in women and first-time parents compared to men and second-time parents. As far as we know there is no previous report on this finding. Even so, it seems to be consistent with proposed theoretical approach identifying (i) the HPA axis as the primary biological mechanism underlying stress regulation and adaptation (Reynolds et al., 2013) and (ii) the role of cortisol, considered the end product of the HPA axis, as a biomarker of psychological stress (Nathanielsz et al., 2003).

Different patterns of change due to gender and parity may indicate cortisol as a stress biomarker for reported higher challenges associated to pregnancy and childbirth for women and first-time parents versus higher demands men and second-time parents related to after childbirth parenting (Colman and Colman, 1991).

The previous hypothesis is consistent with studies analyzing psychological adjustment during pregnancy and early postpartum, when higher anxiety and depression symptoms were found near the end of pregnancy in women and first-time parents and at early postpartum in men and second-time parents, suggesting these periods are the most stressful for each group (e.g., Skari et al., 2002; Twenge et al., 2003; Figueiredo and Conde, 2011). Studies focused on the subjective content of psychological anxiety during pregnancy and early postpartum, involving both women and men (e.g., Matulaitë-Horwood and Bieliauskaitë, 2005), demonstrated that unfinished physical and emotional preparation for the arrival of the new family member and the partner's feelings are identified as the most stressful experiences for men; with delivery (giving birth), anticipated changes in the postpartum period (life after birth), baby's health in birth and afterwards (baby's health), the ability to look after the child and notions of maternal competency are pointed out as the most stressful experiences for women. Additionally, empirical evidence suggests that prior maternal experience may buffer second-time parents from a variety of adverse situations (e.g., fear related with labor and delivery, child's and mother's health) found to influence first-time parents. The effect of parity favoring multiparous women primarily occurs during the first postpartum weeks, before first-time parents acquiring experience. Subsequently, second-time parents seem to be higher disrupted by the need to face multiple responsibilities that need to be addressed, including professional issues and the need to look after the newborn and their other children (for a review see Corter and Fleming, 2002).

Previous studies about cortisol during pregnancy and the postpartum period mainly focused on primiparous women, analyzing cortisol reactivity under stressful situations. Little is known about cortisol changes in men and in second-time parents involving naturalistic, longitudinal designs, which is important due to increased evidence suggesting interindividual variations in HPA axis activity in pregnancy and postpartum. As far as we were able to ascertain, the present study is the first having a longitudinal design following both women and men, first- and second-time parents across pregnancy and early postpartum, and analyzing 24-h urinary free cortisol patterns over the 2 nd and 3rd pregnancy trimesters and 3-months postpartum. This seems to be an innovative perspective in the analysis of course, related factors, and the effects of hormonal changes during pregnancy and the postpartum period.

Findings appear to clarify the direction, as well as, the timing, gender and parity extension of the changes in 24-h 
urinary free cortisol from mid-pregnancy to 3-months postpartum. The same pattern of change in all parents' 24-h urinary free cortisol from mid-pregnancy to 3 -months postpartum is consistent with the role of hormonal changes in gestation and preparation to parenting. Differences on 24$\mathrm{h}$ urinary free cortisol according to gender and parity are consistent with cortisol as a stress biomarker for higher challenges associated to pregnancy and childbirth for women and first-time parents versus higher demands related to after childbirth parenting for men and second-time parents. We suggest that pregnancy and the early postpartum can be compared to a period of chronic stress followed by a dysregulation of the HPA axis, with consequent changes in the 24-h urinary free cortisol levels. If this hypothesis is confirmed, it will support the value of this measure in assessing distress related to developmental tasks inherent to the transition to parenthood, contrary to what has been indicated by previous research studies suggesting that the collection of urinary cortisol does not adequately captures short-term changes in response to acute stressors (Lovallo and Thomas, 2000).

Despite the effect size variation between medium to large magnitude, the small sample size is the major limitation of the present study, implying that the results should be considered with caution. 24-h urinary free cortisol is considered the best matrix to evaluate overall or longterm HPA activity during this period (Yehuda et al., 2003). Some methodological constraints need to be referred. Cortisol measurement validity depends on the compliance with instructions. It also implies the non-occurrence of acute stress-inducing situations at the time of data collection. Both conditions may result in increased measurement error if not properly controlled. Participants' 24-h urinary free cortisol levels are within the reference range expected for the general population.

Parenting involves a complex interplay between physiological and experiential factors that imply the involvement of a considerable number of biologically active hormones that influence each other in an integrated action. Interindividual variations in the HPA axis during pregnancy have mostly been studied in the context of maternal stress, being associated with anxiety/depression symptoms (for a review see Talge et al., 2007). However, several other studies show that stress and anxiety levels assessed by questionnaire do not necessarily correlate with maternal cortisol levels (Harville et al., 2009; Voegtline et al., 2013). The establishment of a hormonal profile during the transition to parenthood and involving larger samples, in order to simultaneously analyze pregnancy and early postpartum psychophysiological adjustment occurring with the couple and taking into account parity is important. This may generate new knowledge about early biological markers of psychological maladjustment and allow the screening of parents at increased risk, even among low risk community populations. The knowledge of gender and parity differences and effects in psychophysiological changes during this period will also contribute towards the establishment of new targets for interventions during pregnancy and postpartum, thus preventing parents' puerperal disorders associated with HPA dysregulation and its impact on the child's outcomes (Duthie and Reynolds, 2013).

Constraints related with experimental research in human beings imply the adoption of different and more indirect methodologies to build knowledge about the psychobiology of events surrounding the transition to parenthood. Findings from previous studies analyzing the negative prediction of infant's outcomes considering maternal cortisol levels pointed out that this prediction seems to be dependent on the type of cortisol measurement, as well as, the timing of sample collection, gestation assessment and whether the total cortisol or free (bioavailable) cortisol is measured (Duthie and Reynolds, 2013). Therefore, the replication of the present study comparing a variety of matrices for cortisol assessment is also suggested, in order to further explore non-consensual results usually found in this domain.

Finally, following a system perspective implying the triad infant-mother-father, we believe this is essential to develop and/or validate theoretical models to explain the role of cortisol in parents' adjustment to the transition to parenthood and its impact on foetal programming, in addition to planning psychological interventions focused in the specific needs of women, men, first- and second-time parents during each stage of pregnancy and postpartum.

\section{Role of the funding source}

This research was funded under a doctoral grant for Science in Measure IV.3, and co-funded under the 2010 Science and Innovation Operational Program (POCI 2010) from Science and Technology Foundation, Government of the Portuguese Republic (ref. SFRH/BD/13768/2003). This was also supported by FEDER Funds through the Programa Operacional Factores de Competitividade - COMPETE and by National Funds through FCT - Fundação para a Ciência e a Tecnologia under the project: PTDC/SAU/SAP/116738/2010 (PI: Bárbara Figueiredo). The sponsors had no further role in the study design; in the collection, analysis and interpretation of data; in the writing of the report; and in the decision to submit the paper for publication.

\section{Conflict of interest}

This work has no conflict of interest.

\section{Acknowledgements}

We would like to thank the women and men who agreed to participate in the present study.

\section{References}

Bayer Diagnostics ADVIA Centaur Reference Manual, Revision D. Bayer Diagnostics ADVIA Centaur Assay Manual, Revision AT.

Brunton, P.J., Russell, J.A., Douglas, A.J., 2008. Adaptive responses of the maternal hypothalamic-pituitary-adrenal axis during pregnancy and lactation. J. Neuroendocrinol. 20, 764-776.

Carter, C.S., 1998. Neuroendocrine perspectives on social attachment and love. Psychoneuroendocrinology 23 (8), 779-818.

Carter, C.S., Porges, S.W., 2012. Neurobiology and the evolution of mammalian social behavior. In: Narvaez, D., Panksepp, J., Schore, A.N., Gleason, T.R. (Eds.), Evolution, Early Experience and Human Development: From Research to Practice and Policy. Oxford University Press, New York, NY, pp. 132-151. 
Cheng, C., Pickler, R.H., 2010. Maternal psychological well-being and salivary cortisol in late pregnancy and early postpartum. Stress Health 26, 215-224.

Colman, L.L., Colman, A.D., 1991. Pregnancy: The Psychological Experience (Rev. and expanded ed.). The Noonday Press, NY.

Corter, C.M., Fleming, A.S., 2002. Psychobiology of maternal behavior in human beings. In: Bornstein, M.H. (Ed.), Handbook of Parenting: Biology and Ecology of Parenting, vol. 2. Lawrence Erlbawm Associates, Publishers, pp. 141-181.

Corwin, E.J., Pajer, K., 2008. The psychoneuroimmunology of postpartum depression. J. Womens Health 17, 1529-1534.

D’Anna-Hernandez, K.L., Ross, R.G., Natvig, C.L., Laudenslager, M.L., 2011. Hair cortisol levels as a retrospective marker of hypothalamic-pituitary axis activity throughout pregnancy: comparison to salivary cortisol. Physiol. Behav. 104, 348-353.

de Weerth, C., Buitelaar, J.K., 2005. Cortisol awakening response in pregnant women. Psychoneuroendocrinology 30, 902-907.

Dipietro, J.A., 2012. Maternal stress in pregnancy: considerations for fetal development. J. Adolesc. Health 51 (2 Suppl.), S3-S8.

Duthie, L., Reynolds, R.M., 2013. Changes in the maternal hypothalamic-pituitary-adrenal axis in pregnancy and postpartum: influences on maternal and fetal outcomes. Neuroendocrinology 98, 106-115.

Field, T., Diego, M., Hernandez-Reif, M., Vera, Y., Gil, K., Schanberg, S., Kuhn, C., Gonzalez-Garcia, A., 2004. Prenatal maternal biochemistry predicts neonatal biochemistry. Int. J. Neurosci. 114, 933-945.

Figueiredo, B., Conde, A., 2011. Anxiety and depression symptoms in women and men from early pregnancy to 3-months postpartum: parity differences and effects. J. Affect. Disord. 132, 146-157.

Figueiredo, B., Costa, R., 2009. Mother's stress, mood and emotional involvement with the infant: 3 months before and 3 months after childbirth. Arch. Womens Ment. Health 12, 143-153.

Fleming, A.S., Steiner, M., Corter, C.M., 1997. Cortisol, hedonics, and maternal responsiveness in human mothers. Horm. Behav. 32 (2), 85-98.

Gonzalez, A., Atkinson, L., Fleming, A.S., 2009. Attachment and the comparative psychobiology of mothering. In: de Haan, M., Gunnar, M.R. (Eds.), Handbook of Developmental Social Neuroscience. Guilford Press, New York, NY, pp. 225-245.

Harville, E.W., Savitz, D.A., Dole, N., Herring, A.H., Thorp, J.M., Light, K.C., 2007. Patterns of salivary cortisol secretion in pregnancy and implications for assessment protocols. Biol. Psychol. 74, 85-91.

Harville, E.W., Savitz, D.A., Dole, N., Herring, A.H., Thorp, J.M., 2009. Stress questionnaires and stress biomarkers during pregnancy. J. Womens Health 18, 1425-1433.

Jensen, E., Wood, C.E., Keller-Wood, M., 2005. Chronic alterations in ovine maternal corticosteroid levels influence uterine blood flow and placental and fetal growth. Am. J. Physiol.: Regul. Integr. Comp. Physiol. 288, R54-R61.

Jones, N.M., Holzman, C.B., Zanella, A.J., Leece, C.M., Rahbar, M.H., 2006. Assessing mid-trimester salivary cortisol levels across three consecutive days in pregnant women using an at-home collection protocol. Paediatr. Perinat. Epidemiol. 20, $425-437$.

Jung, C., Ho, J.T., Torpy, D.J., Rogers, A., Doogue, M., Lewis, J.G., Czajko, R.J., Inder, W.J., 2011. A longitudinal study of plasma and urinary cortisol in pregnancy and postpartum. J. Clin. Endocrinol. Metab. 96, 1533-1540.

Kammerer, M., Taylor, A., Glover, V., 2006. The HPA axis and perinatal depression: a hypothesis. Arch. Womens Ment. Health 9, 187-196.

Kirschbaum, C., Hellhammer, D.H., 1994. Salivary cortisol in psychoneuroendocrine research: recent developments and applications. Psychoneuroendocrinology 19, 313-333.
Kudielka, B.M., Kirschbaum, C., 2005. Sex differences in HPA axis responses to stress: a review. Biol. Psychol. 69, 113-132.

Lazinski, M.J., Shea, A.K., Steiner, M., 2008. Effects of maternal prenatal stress on offspring development: a commentary. Arch. Womens Ment. Health 11, 363-375.

Lovallo, W.R., Thomas, T.L., 2000. Stress hormones in psychophysiological research: emotional, behavioral, and cognitive implications. In: Cacioppo, J.T., Tassinary, L.G., Berntson, G.G. (Eds.), Handbook of Psychophysiology. , 2nd ed. Cambridge University Press, New York, pp. 342-367.

Magiakow, M.A., Mastorakos, G., Rabin, D., Margioris, A.N., Dubbert, B., Calogero, A.E., Tsigos, C., Munson, P.J., Chrousos, G.P., 1996. The maternal hypothalamic-pituitary-adrenal axis in the third trimester of pregnancy. Clin. Endocrinol. (Oxf.) 44, 419-428.

Mastorakos, G., Ilias, I., 2003. Maternal and fetal hypothalamic-pituitary-adrenal axes during pregnancy and postpartum. Ann. N. Y. Acad. Sci. 997, 136-149.

Matulaitë-Horwood, A., Bieliauskaitë, R., 2005. The subjective content of psychological anxiety in the last month of pregnancy. Acta Med. Litu. 12, 31-36.

Meinlschmidt, G., Martin, C., Neumann, I.D., Heinrichs, M., 2010. Maternal cortisol in late pregnancy and hypothalamic-pituitary-adrenal reactivity to psychosocial stress postpartum in women. Stress 13, 163-171.

Nathanielsz, P.W., Berghorn, K.A., Derks, J.B., Giussani, D.A., Docherty, C., Unno, N., Davenport, A., Kutzlers, M., Koenen, S., Visser, G.H., Nijland, M.J., 2003. Life before birth: effects of cortisol on future cardiovascular and metabolic function. Acta Paediatr. 92, 766-772.

Nunes-Costa, R., Figueiredo, B., Moya-Albiol, L., 2015. The state of art of biological processes in paternal care. Psicol.: Reflex. Crít. 28 (1).

O’Keane, V., Lightman, S., Patrick, K., Marsh, M., Papadopoulos, A.S., Pawlby, S., Seneviratne, G., Taylor, A., Moore, R., 2011. Changes in the maternal hypothalamic-pituitary-adrenal axis during the early puerperium may be related to the postpartum blues. J. Neuroendocrinol. 23, 1149-1155.

Obel, C., Hedegaard, M., Henriksen, T.B., Secher, N.J., Olsen, J., Levine, S., 2005. Stress and salivary cortisol during pregnancy. Psychoneuroendocrinology 30, 647-656.

Owens, P.C., Smith, R., Brinsmead, M.W., Hall, C., Rowley, M., Hurt, D., Lovelock, M., Chan, E.C., Cubis, J., Lewin, T., 1987. Postnatal disappearance of the pregnancy associated reduced sensitivity of plasma cortisol to feedback inhibition. Life Sci. 41, 1745-1750.

Reburn, C.J., Wynne-Edwards, K.E., 1999. Hormonal changes in males of a naturally biparental and uniparental mammal. Horm. Behav. 35, 163-176.

Reynolds, R.M., Hii, H.L., Pennell, C.E., McKeague, I.W., Kloet, E.R., Lye, S., Stanley, F.J., Mattes, E., Foster, J.K., 2013. Analysis of baseline hypothalamic-pituitary-adrenal activity in late adolescence reveals gender specific sensitivity of the stress axis. Psychoneuroendocrinology 38, 1271-1280.

Santos, V., Moura, M., Pinho, J.P., Almeida, V., Maio, J., 2011. Características sócio-demográficas das puérperas e seguimento da gravidez: o que mudou em 17 anos? Acta Med. Port. 24, 877-884.

Sarkar, P.L., Zeng, L., Cen, Y., Salvante, K.G., Nepomnaschy, P.A., 2013. A longitudinal evaluation of the relationship between first morning urinary and salivary cortisol. Am. J. Hum. Biol. 25, 351-358.

Skari, H., Skreden, M., Malt, U.F., Dalholt, M., Ostensen, A.B., Egeland, T., Emblem, R., 2002. Comparative levels of psychological distress, stress symptoms, depression and anxiety after childbirth-a prospective population-based study of mothers and fathers. BJOG 109, 1154-1163.

Stallings, J., Fleming, A.S., Corter, C., Worthman, C., Steiner, M., 2001. The effects of infant cries and odors on sympathy, cortisol, 
and autonomic responses in new mothers and nonpostpartum women. Parenting: Sci. Pract. 1, 71-100.

Storey, A.E., Walsh, C.J., Quinton, R., Wynne-Edwards, K.E., 2000. Hormonal correlates and paternal responsiveness in new and expectant fathers. Evol. Hum. Behav. 21, 79-95.

Talge, N.M., Neal, C., Glover, V., 2007. Antenatal maternal stress and long-term effects on child neurodevelopment: how and why? J. Child Psychol. Psychiatry 48, 245-261.

Teixeira, C., Figueiredo, B., Conde, A., Pacheco, A., Costa, R., 2009. Anxiety and depression during pregnancy in women and men. J. Affect. Disord. 119, 142-148.

Tu, M.T., Lupien, S.J., Walker, C.D., 2006. Multiparity reveals the blunting effect of breastfeeding on physiological reactivity to psychological stress. J. Neuroendocrinol. 18, 494-503.

Twenge, J.M., Campbell, W.K., Foster, C.A., 2003. Parenthood and marital satisfaction: a meta-analytic review. J. Marriage Fam. $65,574-583$.
Voegtline, K.M., Costigan, K.A., Kivlighan, K.T., Laudenslager, M.L., Henderson, J.L., DiPietro, J.A., 2013. Concurrent levels of maternal salivary cortisol are unrelated to self-reported psychological measures in low-risk pregnant women. Arch. Womens Ment. Health 16, 101-188.

Wynne-Edwards, K.E., Reburn, C.J., 2000. Behavioral endocrinology of mammalian fatherhood. Trends Ecol. Evol. 15, 464-468.

Yehuda, R., Halligan, S.L., Yang, R.K., Guo, L.S., Makotkine, I., Singh, B., Pickholtz, D., 2003. Relationship between 24-hour urinary-free cortisol excretion and salivary cortisol levels sampled from awakening to bedtime in healthy subjects. Life Sci. 73, 349-358.

Ziegler, T.E., et al., 1996. Hormonal responses to parental and non-parental conditions in male cotton-top tamarins, Saguinus oedipus, a New World primate. Horm. Behav. 30, 287-297. 